\title{
Otimização da extração assistida por ultrassom de compostos bioativos da espécie
}

\section{Caryocar brasiliense}

\author{
Optimization of ultrasound-assisted extraction of bioactive compounds from Caryocar brasiliense \\ Optimización de la extracción asistida por ultrasonido de compuestos bioactivos de Caryocar \\ brasiliense
}

\author{
Rafael Severino da Silva \\ ORCID: https://orcid.org/0000-0002-6176-6662 \\ Universidade Federal de Jataí, Brasil \\ E-mail: faelssilva7@gmail.com \\ Hellen Bertoletti Barbieri \\ ORCID: https://orcid.org/0000-0002-6270-3874 \\ Universidade Federal de Jataí, Brasil \\ E-mail: hellen.hbb@gmail.com \\ Hevelyn Savio Ferreira \\ ORCID: https://orcid.org/0000-0003-2307-0821 \\ Universidade Federal De Jataí, Brasil \\ E-mail: hevelynsavio@hotmail.com \\ Claudinei Alves da Silva \\ ORCID: https://orcid.org/0000-0003-2442-031X \\ Universidade Federal de Jataí, Brasil \\ E-mail: clalvess@ufj.edu.br \\ Liliane Nebo \\ ORCID: https://orcid.org/0000-0002-5182-7003 \\ Universidade Federal de Jataí, Brasil \\ E-mail: liliane_nebo@ufj.edu.br
}

\begin{abstract}
Resumo
Caryocar brasiliense, popularmente conhecido como pequi, é uma espécie que apresenta alto teor de compostos fenólicos com propriedades biológicas. Neste trabalho, o processo de extração assistida por ultrassom, de compostos bioativos das folhas do pequi, foi otimizado com planejamento fatorial Box-Behnken. Quatro variáveis independentes foram avaliadas: concentração da amostra (relação:líquido-sólido (L-S), proporção de etanol na solução $\left(\mathrm{EtOH} / \mathrm{H}_{2} \mathrm{O}\right.$ $\%$ v/v), tempo de EAU (extração assistida por ultrassom) e temperatura. A metodologia de Superfície de Resposta foi usada para apontar a variável mais importante no processo. Os resultados revelaram que a proporção EtOH/ $\mathrm{H}_{2} \mathrm{O}(\%$ $\mathrm{v} / \mathrm{v})$ tem o efeito mais significante na extração dos compostos fenólicos. A condição ótima para extração foi de etanol $60 \%$, tempo de EAU de 30 min, temperatura da EAU de $42{ }^{\circ} \mathrm{C}$ e L-S 18,7:1. Sob essas condições foram extraídos $6,30 \mathrm{mg}$ EAG/g ES $(\mathrm{n}=3)$ ( $\mathrm{mg}$ de equivalente de ácido gálico/ $\mathrm{g}$ de extrato seco). Os valores experimentais observados confirmam a adequação do modelo empregado e seu sucesso para otimização das condições de extração.
\end{abstract}

Palavras-chave: Extrato das folhas; Box-Behnken; Compostos fenólicos; Pequi.

\begin{abstract}
Caryocar brasiliense, popularly known as pequi, is a species that has a high content of phenolic compounds with biological activities. In this work, the ultrasound-assisted extraction process of bioactive compounds of the pequi leaves was optimized with Box-Behnken factorial design. Four independent variables were investigated: sample concentration (liquid-solid ratio, LS), ethanol concentration $\left(\mathrm{EtOH} / \mathrm{H}_{2} \mathrm{O} \% \mathrm{v} / \mathrm{v}\right.$ ), UAE time (ultrasound-assisted extraction) and UAE temperature. The Response Surface methodology was used to point out the most important variable in the process. The results revealed the $\mathrm{EtOH} / \mathrm{H}_{2} \mathrm{O}(\% \mathrm{v} / \mathrm{v})$ the most significant effect on the extraction of phenolic compounds. The optimum condition for extraction was an ethanol concentration of $60 \%$, UAE time of 30 min, a UAE temperature of $42{ }^{\circ} \mathrm{C}$, and LS 18.7: 1 . Under these conditions $6.30 \mathrm{mg}$ GAE/g DW $(\mathrm{n}=3)$ were extracted ( $\mathrm{mg}$ gallic acid equivalent /g of dry weight). The observed experimental results confirm the suitability of the model used and its success for the optimization of extraction conditions.
\end{abstract}

Keywords: Leaves extracts; Box-Behnken; Phenolic compounds; Pequi.

\section{Resumen}

Caryocar brasiliense, popularmente conocido como pequi, es una especie que tiene un alto contenido de compuestos fenólicos con propiedades biológicas. En este trabajo, se optimizó el proceso de extracción asistida por ultrasonido de 
compuestos bioactivos de hojas de pequi con el diseño factorial de Box-Behnken. Se evaluaron cuatro variables independientes: concentración de la muestra (relación: líquido-sólido (LS), proporción de etanol en la solución $\left(\mathrm{EtOH} / \mathrm{H}_{2} \mathrm{O} \% \mathrm{v} / \mathrm{v}\right.$ ), tiempo UAE (extracción asistida por ultrasonido) y temperatura. Se utilizó la metodología Response Surface para señalar la variable más importante en el proceso. Los resultados revelaron que la proporción $\mathrm{EtOH} / \mathrm{H}_{2} \mathrm{O}(\% \mathrm{v} / \mathrm{v})$ tiene el efecto más significativo en la extracción de compuestos fenólicos. La condición óptima para la extracción fue $60 \%$ de etanol, tiempo de EAU $30 \mathrm{~min}$, temperatura de EAU de $42{ }^{\circ} \mathrm{C}$ y L-S 18.7: 1. En estas condiciones se extrajeron $6,30 \mathrm{mg}$ EAG/g ES $(\mathrm{n}=3)(\mathrm{mg}$ de equivalente de ácido gálico/g extracto seco). Los valores experimentales observados confirman la adecuación del modelo empleado y su éxito en la optimización de las condiciones de extracción.

Palabras clave: Extractos de hojas; Box-Behnken; Compuestos fenólico; Pequi.

\section{Introdução}

O Brasil é um país rico em fauna e flora, presentes em seus biomas, como o bioma do Cerrado se destaca por ser o segundo maior bioma do país e apresentar uma grande diversidade de espécies de plantas, possuindo inúmeras atividades biológicas (Neto, et al., 2020). Dentre as espécies presentes no Cerrado, destaca-se a Caryocar brasiliense, conhecido popularmente como pequi, pertence à família Caryocaraceae no qual abrange cerca de 16 espécies encontradas no território brasileiro (de Almeida, et al., 2019; de Oliveira, et al., 2018). Seu fruto é bastante empregado na culinária brasileira. Contudo, essa espécie não se limita apenas como fonte de alimento, observa-se na literatura várias atividades biológicas, dentre elas, antiviral, antiedematogênica, anti-inflamatória, antitumoral, antimicrobiana e antioxidante (Baptista, et al., 2018; Bemfeito, et al., 2020; Caldeira, et al., 2021; Junior, et al., 2019; Nascimento-Silva \& Naves, 2019). Estudos correlacionados à C. brasiliense demonstram um alto teor de compostos com atividade antioxidante (Fonseca, et al., 2018; Nascimento-Silva, et al., 2020; Ramos, et al., 2021). Assim, tanto a espécie quanto seus metabólitos são de grande interesse, principalmente no ramo farmacológico (Oliveira, et al., 2020; Pires, et al., 2020).

Aliado aos estudos de compostos bioativos, observa-se também, um grande crescimento no desenvolvimento de novos métodos otimizados e/ou padronizados que buscam aprimorar os processos de extração de compostos bioativos (Ahmad, et al., 2020; Altemimi, et al., 2020; Valu, et al., 2020). Dentre as metodologias analíticas baseadas nas técnicas de extração que, visam minimizar a geração de resíduos de solventes orgânicos e que permitem recuperações mais eficientes de compostos bioativos a partir de matrizes vegetais, destaca-se a extração assistida por ultrassom (Vizzotto \& Pereira, 2011). Essa técnica é conhecida por acelerar o processo de extração por meio de ondas de baix a frequência resultando em cavitações que facilitam na penetração do solvente nas paredes celulares da matriz vegetal (Ghitescu, et al., 2015).

A otimização de um processo de extração visa avaliar as melhores condições de um sistema para extrair determinado analito (Güray, et al., 2020; Nam, et al., 2017). No processo de extração via ultrassom os possíveis parâmetros que influenciam na extração de metabólitos secundários são: concentração da amostra, proporção de solvente, tempo de extração e temperatura (Ghitescu, et al., 2015). Desse modo, a utilização de ferramentas estatísticas, tais como o planejamento fatorial e a superfície de resposta, vêm sendo empregadas, a fim de explorar condições experimentais de sistemas com um menor número de análises (El-Naggar, et al., 2021; Yuan, et al., 2018; Zhang, et al., 2021). O planejamento Box-Behnken por exemplo, é uma metodologia que utiliza tais ferramentas para o auxílio na definição de quais as melhores condições para extração dos analitos de interesse. Estudos correlacionados a este método têm mostrado uma grande eficiência na extração de compostos bioativos presentes em diversas espécies botânicas (Jiang, et al., 2020; Moon, et al., 2020). Nesse sentido, esse trabalho empregou o planejamento Box-Behnken como ferramenta estatística para otimizar o processo de extração de compostos fenólicos das folhas da espécie C. brasiliense via extração assistida por ultrassom. 


\section{Metodologia}

\subsection{Coleta da espécie}

As folhas de Caryocar brasiliense foram coletadas no campus Jatobá da Universidade Federal de Jatai (UFJ), Jataí-GO sob voucher HJ 8234 (Herbário Jataiense/UFJ).

Após a coleta, as folhas foram lavadas com água destilada, limpas e secas à $40{ }^{\circ} \mathrm{C}$ na estufa de circulação forçada de ar (Quimis - Q314M) por 4 dias e, posteriormente trituradas em liquidificador industrial.

\subsection{Instrumentação e Reagentes}

Os equipamentos Espectrofotômetro UV-VIS Femto - Espectrofotômetro 700 Plus (Faixa:195 a 1100 nm; Resolução: 1 nm); Aparelho de ultrassom Unique - USC 2500 e Centrífuga refrigerada NovaTecnica - NT 825 Centrífuga Refrigerada utilizados neste trabalho encontram-se no Laboratório de Química Geral e no Laboratório de Química Orgânica e Inorgânica da Universidade Federal de Jataí.

Solventes utilizados no processo de extração: Etanol (96 \% Grau/PA), água destilada, reagente de Folin-Ciocalteu, $\mathrm{Na}_{2} \mathrm{CO}_{3}$ e outros reagentes da Marca Vetec.

\subsection{Planejamento Experimental - Box-Behnken}

\subsubsection{Otimização do Fator único}

Para avaliar a influência da relação líquido-sólido (L-S), concentração de etanol (EtOH/ $\mathrm{H}_{2} \mathrm{O} \%$ v/v), tempo de extração assistida por ultrassom (EAU) e temperatura de EAU na extração de fenólicos, utilizou-se, para cada ensaio, 1,00 g folhas de C. brasiliense, seguindo os níveis propostos na tabela 1 e os seguintes tópicos abaixo.

- Para a variação da relação Líquido-Sólido, manteve-se constante a proporção de 80 \% EtOH, 50 min de EAU e temperatura a $40{ }^{\circ} \mathrm{C}$.

- Para a variação da proporção de EtOH na solução, manteve-se constante a relação Líquido-Sólido de 15:1, 50 min de EAU e temperatura a $40{ }^{\circ} \mathrm{C}$.

- Para a variação do tempo de EAU, manteve-se constante a relação Líquido-Sólido de 15:1, proporção de 80 \% EtOH e temperatura a $40{ }^{\circ} \mathrm{C}$.

- Para a variação da temperatura, manteve-se constante a relação Líquido-Sólido de 15:1, proporção de 80 \% EtOH e 50 min de EAU.

Tabela 1 - Variáveis propostas para obtenção do fator único.

\begin{tabular}{c|c|c|c|c|c}
\hline \hline \multicolumn{5}{c}{ Planejamento fator único } \\
\hline Variáveis & Codificação & \multicolumn{4}{c}{ Níveis } \\
\hline \hline Líquido-Sólido $(\mathbf{m L} / \mathbf{g})$ & $(\mathbf{A})$ & $10: 1$ & $15: 1$ & $20: 1$ & $25: 1$ \\
\hline EtOH/H2O $(\% \mathbf{~ v / v ) ~}$ & $($ B) & 60 & 70 & 80 & 90 \\
\hline Temperatura $\left({ }^{\circ} \mathbf{C}\right)$ & $(\mathbf{C})$ & 30 & 40 & 50 & 60 \\
\hline Tempo de EAU $(\mathbf{m i n})$ & (D) & 20 & 30 & 40 & 50 \\
\hline \hline
\end{tabular}

Fonte: Autores.

Após ensaios, os extratos foram centrifugados por $10 \mathrm{~min}$ na rotação de $4000 \mathrm{rpm}$. Por fim, retirou-se a fase líquida de cada tubo Falcon e realizou-se os ensaios de quantificação dos compostos fenólicos totais. Todas as extrações foram realizadas em triplicata. 


\subsubsection{Planejamento Fatorial - Box-Behnken}

A partir dos resultados obtidos das variáveis de otimização do fator único, foi realizado o planejamento Box-Behnken com as quatro variáveis propostas: concentração da amostra (relação: líquido-sólido (L-S)), concentração de etanol (EtOH/ $\mathrm{H}_{2} \mathrm{O}$ $\%$ v/v), tempo de extração assistida por ultrassom (EAU) e temperatura de EAU.

A realização desse planejamento consistiu em 29 ensaios, no qual foram contempladas cinco réplicas de pontos centrais (0), e os demais variáveis codificadas como (+1) e (-1), sendo (0) corresponde ao ponto de maior teor de compostos fenólicos, de acordo com o resultado fator único, (+1) e (-1) os valores correspondentes aos níveis superior e inferior, respectivamente. Para cada ensaio foi utilizado $1,00 \mathrm{~g}$ folhas seca de $C$. brasiliense, seguindo as codificações propostas e uma ordem experimental aleatória foi usada para reduzir o efeito da variabilidade inexplicada na resposta observada.

\subsubsection{Superfície de Resposta}

Para obter as superfícies de respostas das variáveis foi utilizada uma equação polinomial de segunda ordem:

$$
\begin{aligned}
Y= & b_{0}+b_{1} A+b_{2} B+b_{3} C+b_{4} D+b_{12} B A+b_{13} A C+b_{14} A D+ \\
& b_{23} B C+b_{24} B D+b_{34} C D+b_{11} A^{2}+b_{22} B^{2}+b_{33} C^{2}+b_{44} D^{2}
\end{aligned}
$$

Onde $\mathrm{Y}$ corresponde ao teor de compostos fenólicos, $\mathrm{b}_{0}, \mathrm{~b}_{1}, \mathrm{~b}_{2}, \mathrm{~b}_{3}, \mathrm{~b}_{4}, \mathrm{~b}_{11}, \mathrm{~b}_{22}$, ... são os coeficientes de regressão; e A, B, C e D são fatores independentes para a relação: líquido-sólido, concentração de etanol $\left(\mathrm{EtOH} / \mathrm{H}_{2} \mathrm{O} \%\right.$ v/v), tempo de extração assistida por ultrassom (EAU) e temperatura de EAU.

Empregou-se análise de variância (ANOVA) frente aos dados obtidos na superfície de resposta com intuito de determinar falhas de ajustes e efeitos das variáveis provenientes de interações relacionadas ao teor de compostos fenólicos. Para análise estatística, utilizou-se o programa Design-Expert ${ }^{\circledR} 11.0$ (Versão Teste) considerando o percentual de compostos fenólicos totais como resposta com intervalo de confiança de $95 \%$.

\subsection{Quantificação de fenóis totais}

A quantificação de fenóis totais foi realizada por meio do método de Folin-Ciocalteu segundo Singleton, et al. (1999). Soluções etanólicas dos extratos na concentração de $250 \mu \mathrm{g} \cdot \mathrm{mL}^{-1}$ foram preparadas e dessas, para cada ensaio, $100 \mu \mathrm{L}$ foram adicionados a $500 \mu \mathrm{L}$ do reagente de Folin-Ciocalteu, $6,40 \mathrm{~mL}$ de água destilada e $2,00 \mathrm{~mL}$ de $\mathrm{Na}_{2} \mathrm{CO}_{3}$ a $15 \%(\mathrm{~m} / \mathrm{m})$, agitada por 60 segundos e colocada em repouso em local fechado, sem a presença de qualquer tipo de luz.

Após 1 hora, a absorbância das amostras foi medida a $760 \mathrm{~nm}$ em espectrômetro de UV-Vis. A análise de um branco da amostra também foi realizada em acordo com o método. O teor de fenóis totais foi determinado nas amostras por meio da curva de calibração, utilizando o ácido gálico $\left(15,0\right.$ a $\left.500 \mu \mathrm{g} \cdot \mathrm{mL}^{-1}\right)$ como referência, e expressos como miligrama de EAG (equivalentes de ácido gálico) por g de extrato seco (mg (EAG)/g ES). Todas as análises foram realizadas em triplicata.

\subsubsection{Limite de Deteç̧ão e Quantificação (LOD E LOQ)}

Os limites de detecção (LOD) e quantificação (LOQ) calculados a partir das equações 1 e 2, respectivamente (Swartz, et al., 1998).

$$
L O D=\frac{3,3 s}{\beta} \quad \text { (eq. 1) LOQ }=\frac{10 s}{\beta} \quad \text { (eq. 2) }
$$

No qual S e $\beta$ correspondem ao valor do desvio padrão da reta e inclinação da curva de calibração, respectivamente. 


\section{Resultados e Discussão}

\subsection{Quantificação dos compostos fenólicos totais}

Os teores de compostos fenólicos foram obtidos a partir da curva de calibração y $=0,0012 \mathrm{x}+0,0408$, medido na absorbância de $760 \mathrm{~nm}$ com $\mathrm{R}^{2}=0,9971$, utilizando o ácido gálico como padrão. Também foram obtidos os valores de limite de quantificação e detecção de $0,893 \mathrm{mg}(\mathrm{EAG}) \cdot \mathrm{g}^{-1}$ e $0,295 \mathrm{mg}(\mathrm{EAG}) \cdot \mathrm{g}^{-1}$, respectivamente.

\subsubsection{Otimização do fator único}

Inicialmente, elegeu-se quatro variáveis que poderiam influenciar no processo de extração dos compostos fenólicos: relação líquido-sólido (L-S), concentração de etanol $\left(\%\right.$ EtOH/ $\left.\mathrm{H}_{2} \mathrm{O}\right)$, tempo de extração assistida por ultrassom (EAU) e temperatura de EAU.

A otimização do fator único foi realizada com o intuito de determinar, inicialmente, os níveis de trabalho, no planejamento Box-Behnken, para as variáveis de escolha por meio das respostas frente a quantidade de compostos fenólicos extraídos por ensaio.

Analisando a Figura 1A, observou-se que as menores proporções (relação líquido/sólido: 10:1 e 15:1) menores teores de fenólicos eram extraídos. Porém, um aumento crescente foi observado a partir da relação líquido/sólido: 20:1. Já para a Figura 1B, pode-se verificar um comportamento linear entre 60 a $90 \%$ de EtOH.

Na Figura 1C, que demonstra o efeito do tempo de extração assistida por ultrassom (EAU), observou-se um aumento no teor de fenólicos entre os pontos 20 a 40min. e um decréscimo quando ultrapassado o tempo de 40min.

Por fim, na Figura 1D, onde observa-se o efeito da temperatura no processo de extração, uma maior eficiência foi observada para a temperatura de $40^{\circ} \mathrm{C}$, seguido pelo decréscimo em temperaturas maiores.

Figura 1 - Efeito das variáveis no processo de extração: A) Relação líquido-sólido. B) Concentração de etanol. C) Tempo de extração assistida por ultrassom. D) Temperatura no ultrassom.

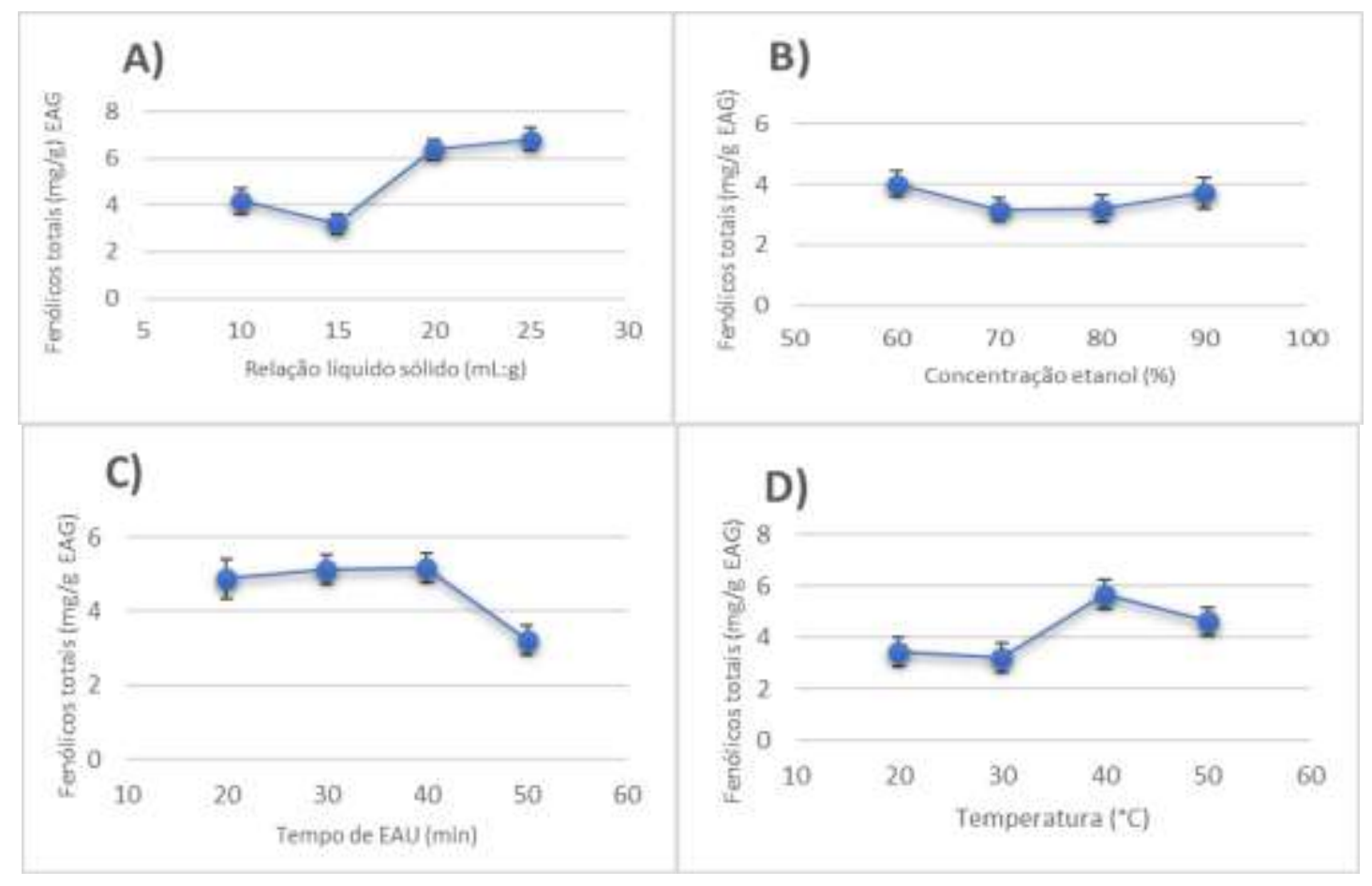

Fonte: Autores. 
Analisando os resultados correlacionados as quatro variáveis, propôs-se utilizar como pontos centrais no planejamento Box-Behnken os seguintes valores: 20:1 relação líquido-sólido, $70 \% \mathrm{EtOH} / \mathrm{H}_{2} \mathrm{O}, 30$ minutos de EAU e $40^{\circ} \mathrm{C}$ (Tabela 2).

\subsubsection{Planejamento fatorial Box-behnken}

A partir dos resultados obtidos na otimização do fator único, foram propostos os pontos centrais, níveis superiores e inferiores para as quatro variáveis (Tabela 2).

Tabela 2 - Níveis de variáveis obtidas através do método de otimização.

\begin{tabular}{lc|c|c}
\hline \hline \multirow{2}{*}{ Variáveis } & \multicolumn{3}{c}{ Níveis } \\
& $\mathbf{- 1}$ & $\mathbf{0}$ & $\mathbf{+ 1}$ \\
\hline \hline Líquido-Sólido & $15: 1$ & $20: 1$ & $25: 1$ \\
\hline Concentração \% EtOH /H20 & 60 & 70 & 80 \\
\hline Tempo EAU & 20 & 30 & 40 \\
\hline Temperatura EAU & 30 & 40 & 50 \\
\hline \hline
\end{tabular}

Fonte: Autores.

O planejamento Box-Behnken foi realizado em 29 ensaios, sendo 5 pontos centrais $(0,0,0,0)$ e os demais pontos com diferentes combinações entre níveis superior, inferior e ponto central. As respostas foram obtidas em função dos teores de fenólicos conforme a Tabela 3. 
Tabela 3 - Planejamento Box-Behnken.

\begin{tabular}{|c|c|c|c|c|c|}
\hline \multirow[b]{2}{*}{ Amostra } & \multicolumn{4}{|c|}{ Variável proposta } & \multirow{2}{*}{$\begin{array}{c}\text { Resposta } \\
\text { [ ] Fenólicos / } \\
\text { mg (EAG).g-1 } \\
\text { ES } *\end{array}$} \\
\hline & $\begin{array}{l}\text { Índice Líquido - } \\
\text { Sólido (A) / mL:g }\end{array}$ & $\begin{array}{l}\text { Concentração } \\
\operatorname{EtOH}(\mathbf{B}) / \%\end{array}$ & $\begin{array}{l}\text { Tempo EAU } \\
\text { (C) / min }\end{array}$ & $\begin{array}{l}\text { Temperatura } \\
\text { EAU (D) } /{ }^{\circ} \mathbf{C}\end{array}$ & \\
\hline 1 & $0(20: 1)$ & (0 (70) & $-1(20)$ & $-1(30)$ & $5,5,09$ \\
\hline 2 & $0(20: 1)$ & $-1(60)$ & $+1(40)$ & $0(40)$ & 6,65 \\
\hline 3 & $0(20: 1)$ & $+1(80)$ & $0(30)$ & $-1(30)$ & 2,50 \\
\hline 4 & $0(20: 1)$ & $0(70)$ & $0(30)$ & $0(40)$ & 5,62 \\
\hline 5 & $0(20: 1)$ & $-1(60)$ & $0(30)$ & $+1(50)$ & 5,39 \\
\hline 6 & $-1(15: 1)$ & $-1(60)$ & $0(30)$ & $0(40)$ & 6,30 \\
\hline 7 & $+1(25: 1)$ & $0(70)$ & $-1(20)$ & $0(40)$ & 3,84 \\
\hline 8 & $0(20: 1)$ & $0(70)$ & $0(30)$ & $0(40)$ & 5,84 \\
\hline 9 & $-1(15: 1)$ & $+1(80)$ & $0(30)$ & $0(40)$ & 3,14 \\
\hline 10 & $0(20: 1)$ & $0(70)$ & $+1(40)$ & $-1(30)$ & 5,52 \\
\hline 11 & $0(20: 1)$ & $0(70)$ & $0(30)$ & $0(40)$ & 6,42 \\
\hline 12 & $0(20: 1)$ & $+1(80)$ & $+1(40)$ & $0(40)$ & 2,32 \\
\hline 13 & $-1(15: 1)$ & $0(70)$ & $0(30)$ & $-1(30)$ & 5,32 \\
\hline 14 & $-1(15: 1)$ & $0(70)$ & $0(30)$ & $+1(50)$ & 4,09 \\
\hline 15 & $0(20: 1)$ & $-1(60)$ & $0(30)$ & $-1(30)$ & 6,02 \\
\hline 16 & $+1(25: 1)$ & $0(70)$ & $0(30)$ & $+1(50)$ & 4,67 \\
\hline 17 & $0(20: 1)$ & $+1(80)$ & $-1(20)$ & $0(40)$ & 2,32 \\
\hline 18 & $0(20: 1)$ & $0(70)$ & $+1(40)$ & $+1(50)$ & 5,82 \\
\hline 19 & $+1(25: 1)$ & $+1(80)$ & $0(30)$ & $0(40)$ & 3,95 \\
\hline 20 & $-1(15: 1)$ & $0(70)$ & $-1(20)$ & $0(40)$ & 5,37 \\
\hline 21 & $+1(25: 1)$ & $0(70)$ & $+1(40)$ & $0(40)$ & 3,99 \\
\hline 22 & $0(20: 1)$ & $0(70)$ & $0(30)$ & $0(40)$ & 6,00 \\
\hline 23 & $0(20: 1)$ & $-1(60)$ & $-1(20)$ & $0(40)$ & 5,95 \\
\hline 24 & $+1(25: 1)$ & $-1(60)$ & $0(30)$ & $0(40)$ & 6,25 \\
\hline 25 & $+1(25: 1)$ & $0(70)$ & $+1(40)$ & $0(40)$ & 4,50 \\
\hline 26 & $+1(25: 1)$ & $0(70)$ & $0(30)$ & $-1(30)$ & 2,69 \\
\hline 27 & $0(20: 1)$ & $0(70)$ & $0(30)$ & $0(40)$ & 6,64 \\
\hline 28 & $0(20: 1)$ & $0(70)$ & $+1(40)$ & $+1(50)$ & 5,12 \\
\hline 29 & $0(20: 1)$ & $+1(80)$ & $0(30)$ & $+1(50)$ & 3,30 \\
\hline
\end{tabular}

*ES: extrato seco. Fonte: Autores.

Os resultados da análise estatística ANOVA (Tabela 4) estão associados aos dados da Tabela 3, empregando o uso do software Desing-Expert ${ }^{\circledR} 7.0$. 
Research, Society and Development, v. 10, n. 9, e41310916493, 2021

(CC BY 4.0) | ISSN 2525-3409 | DOI: http://dx.doi.org/10.33448/rsd-v10i9.16493

Tabela 4 - Análise de variância (ANOVA) para o modo polinomial quadrático.

\begin{tabular}{|c|c|c|c|c|c|}
\hline Fonte & Soma dos Quadrados & Df & Quadrado Médio & Valor F & Valor-P Prob > F \\
\hline Modelo & 41.55 & 14 & 2.97 & 6.73 & 0.0005 \\
\hline A (Liq.-Sol.) & 0.92 & 1 & 0.92 & 2.09 & 0.1698 \\
\hline $\mathrm{B}(\mathrm{EtOH})$ & 30.18 & 1 & 30.18 & 68.41 & $<0.0001$ \\
\hline C (Tempo EAU) & 0.080 & 1 & 0.080 & 0.18 & 0.6766 \\
\hline D (Temperatura) & 0.31 & 1 & 0.31 & 0.71 & 0.4133 \\
\hline $\mathrm{AB}$ & 0.18 & 1 & 0.18 & 0.42 & 0.5278 \\
\hline $\mathrm{AC}$ & 0.26 & 1 & 0.26 & 0.59 & 0.4553 \\
\hline $\mathrm{AD}$ & 2.58 & 1 & 2.58 & 5.84 & 0.0299 \\
\hline $\mathrm{BC}$ & 0.12 & 1 & 0.12 & 0.28 & 0.6065 \\
\hline $\mathrm{BD}$ & 0.51 & 1 & 0.51 & 1.16 & 0.2999 \\
\hline $\mathrm{CD}$ & 0.048 & 1 & 0.048 & 0.11 & 0.7454 \\
\hline$A^{2}$ & 3.02 & 1 & 3.02 & 6.85 & 0.0203 \\
\hline $\mathrm{B}^{2}$ & 3.04 & 1 & 3.04 & 6.90 & 0.0199 \\
\hline$C^{2}$ & 1.44 & 1 & 1.44 & 3.26 & 0.0923 \\
\hline $\mathrm{D}^{2}$ & 2.27 & 1 & 2.27 & 5.14 & 0.0398 \\
\hline Residual & 6.18 & 14 & 0.44 & & \\
\hline Falta de ajuste & 5.88 & 10 & 0.59 & 8.00 & 0.0299 \\
\hline Erro puro & 0.29 & 4 & 0.074 & & \\
\hline Cor Total & 47.73 & 28 & & & \\
\hline $\mathrm{R}^{2}$ & 0.8706 & & & & \\
\hline Adj. $\mathrm{R}^{2}$ & 0.7412 & & & & \\
\hline Pred. $\mathrm{R}^{2}$ & 0.2805 & & & & \\
\hline Adeq Precision & 8.567 & & & & \\
\hline
\end{tabular}

$* \mathrm{P}<0,01$ altamente significativo; $0,01<\mathrm{P}<0,05$ significativo; $\mathrm{P}>0,05$ não significativo. Fonte: Autores.

Os resultados da ANOVA mostram um valor F para o modelo é baixo valor de P (0.0005), revelando que o modelo é significativo. Os valores do coeficiente de determinação $\left(\mathrm{R}^{2}\right.$ - 0,8706) e o valor ajustado do coeficiente de determinação (Adj. $\mathrm{R}^{2}$ - 0,7414) também confirmam que o modelo é adequado. Valores superioes a $80 \%$ são considerados bons valores de coeficientes de determinação.

Por meio da análise estatística dos dados foi possível observar que a variável B, correlacionada ao efeito da concentração de etanol, foi a mais significativa $(\mathrm{P}<0.0001)$ para a extração de compostos fenólicos, já as demais variáveis não tiveram efeitos individualmente significativos no conteúdo de compostos fenólicos extraídos.

Juntamente com a tabela de valores da ANOVA obtida pelo programa Design-Expert ${ }^{\circledR}$ 11.0, foi também obtido uma equação polinomial de segunda ordem que relaciona variáveis independentes e a resposta (Eq. 3).

Fenóis $=5.77-0.28 \mathrm{~A}-1.59 \mathrm{~B}-0.082 \mathrm{C}+0.16 \mathrm{D}+0.22 \mathrm{BA}+0.26 \mathrm{AC}+$ $0.80 \mathrm{AD}-0.18 \mathrm{BC}+0.36 \mathrm{BD}-0.11 \mathrm{CD}-0.68 \mathrm{~A}^{2}-0.68 \mathrm{~B}^{2}-0.47 \mathrm{C}^{2}-0.59 \mathrm{D}^{2} \quad$ (Eq. 3$)$ 


\subsubsection{Análise da superfície de resposta}

A resposta das variáveis dependentes e independentes obtidas na etapa de otimização do processo extrativo são observadas através de gráficos de superfície de respostas tridimensionais (Figura 2A-2F). A resposta dos gráficos se dá em função do teor de compostos fenólicos obtidos através da análise em níveis variados de dois fatores, enquanto os demais fatores foram mantidos constantes em seu respectivo nível zero. As formas de plotagens no formato de contorno, elípticas ou circulares, são os indicativos das interações entre as correspondentes variáveis, mostrando ser significativas.

Na Figura 2A observou-se a variação na concentração de etanol e a relação líquido-sólido, mantendo constante a temperatura em $40^{\circ} \mathrm{C}$ e o tempo de EAU $30 \mathrm{~min}$. A interação entre o efeito destas variáveis mostrou não ser significativa, devido ao valor $\mathrm{P}$ ser maior que 0,05 ( $\mathrm{P}>0,05)$. Analisando a superfície de resposta, nota-se que a diminuição na proporção de EtOH proporciona um aumento no teor de fenólicos extraídos da amostra, o mesmo comportamento se dá em relação a variável líquido-sólido. É observado que a proporção de EtOH/ $\mathrm{H}_{2} \mathrm{O}$ influencia diretamente na extração de compostos fenólicos.

Alguns autores correlacionam que um único solvente muitas vezes, não é capaz de realizar uma extração com valores de rendimentos eficazes, para isso a junção de dois solventes, tais como EtOH/ $\mathrm{H}_{2} \mathrm{O}$ promovem interações moleculares diante dos compostos presentes na espécie botânica auxiliando assim no processo de extração (Lu, et al., 2015; Ez Zoubi, et al., 2021).

$\mathrm{O}$ efeito das váriaveis de tempo de EAU e relação líquido-sólido, mantendo constante o teor de etanol em $70 \%$ e temperatura $40^{\circ} \mathrm{C}$ pode ser observado na Figura $2 \mathrm{~B}$. A interação entre essas duas váriaveis mostrou não ser significativa $(\mathrm{P}$ $>0,05)$. Analisando individualemnte as váriaveis, nota-se: aumento no teor de compostos fenólicos com a variação de 20 para 30min no tempo de EAU, em relação a váriavel líquido-sólido, houve um aumento no teor de compostos fenólicos à medida que aumentou-se a proporção de 15:1 a 17,5:1, porém observou-se um decréscimo no teor de fenólicos a partir da relação váriavel líquido-sólido 17,5:1. O aumento da relação líquido-sólido de 15:1 a 17,5:1, fez com que o solvente conseguisse maior permeabilidade permitindo assim uma maior extração, esse comportamento também foi observado no estudo apresentado por Prasad et al. (2009). 
Figura 2 - Superfície de resposta para os efeitos entre as variáveis.

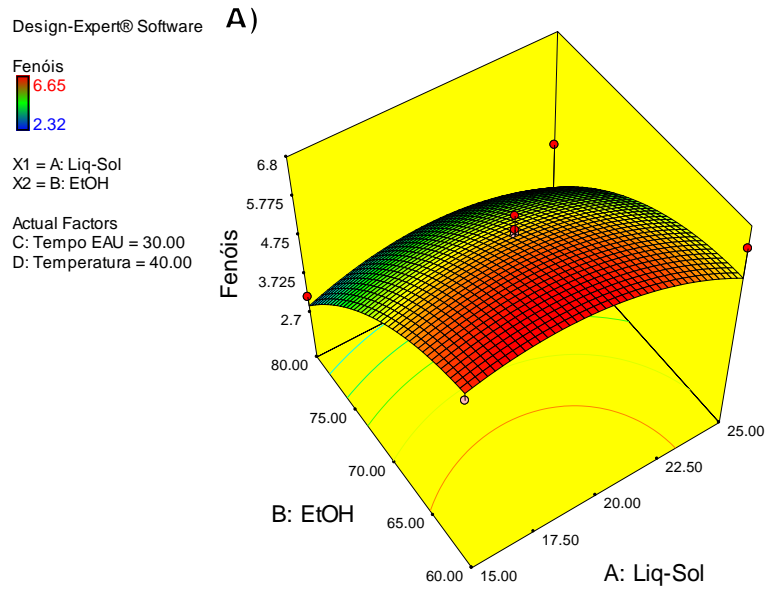

C)

Design-Expert@ Softwa

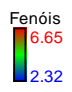

X1 = A: Liq-Sol $\mathrm{X} 2=\mathrm{D}:$ Temperatura Actual Factors B: $E \mathrm{EOH}=70.00$

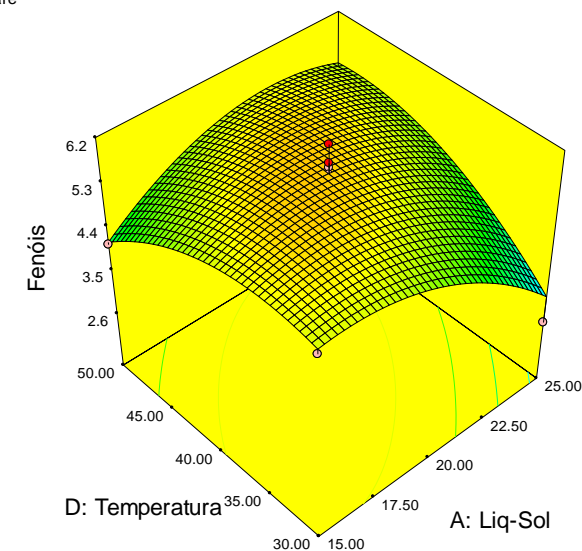

E)

Design-Exper!® Softwa

$\prod_{2.32}^{6.65}$

\section{$\mathrm{X} 1=\mathrm{B}: \mathrm{EtOH}$ $\mathrm{X} 2=\mathrm{D}:$ Temperatura} Actual Factors
A: Liq-Sol $=20.00$ C: Tempo $E A U=30.00$

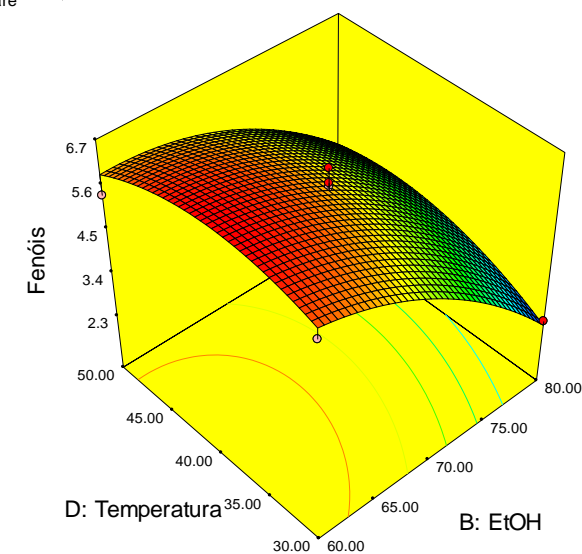

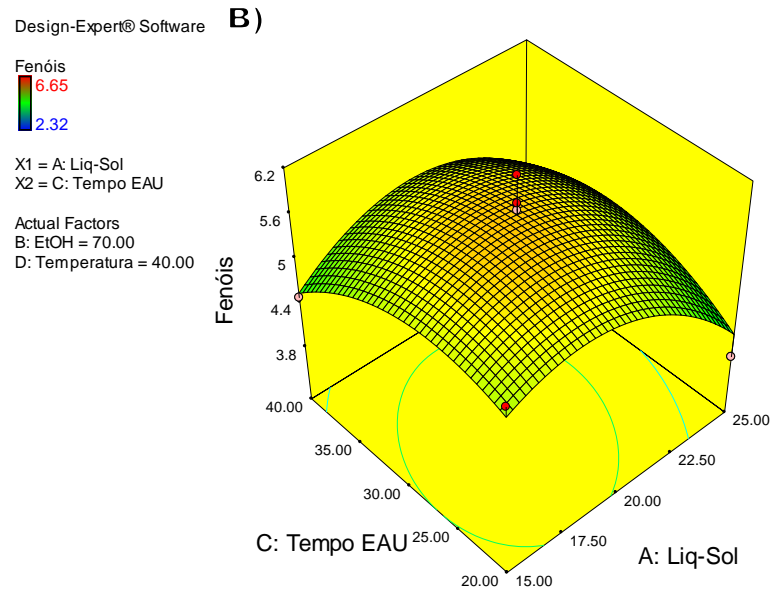

D)

Design-Expert@ Software

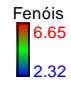

$\mathrm{X} 1=\mathrm{B}: \mathrm{EtOH}$ $\mathrm{X} 1=\mathrm{B}:$ EtOH
$\mathrm{X} 2=\mathrm{C}:$ Tempo EAU Actual Factors A: Liq-Sol $=20.00$ Temperatura $=40.00$

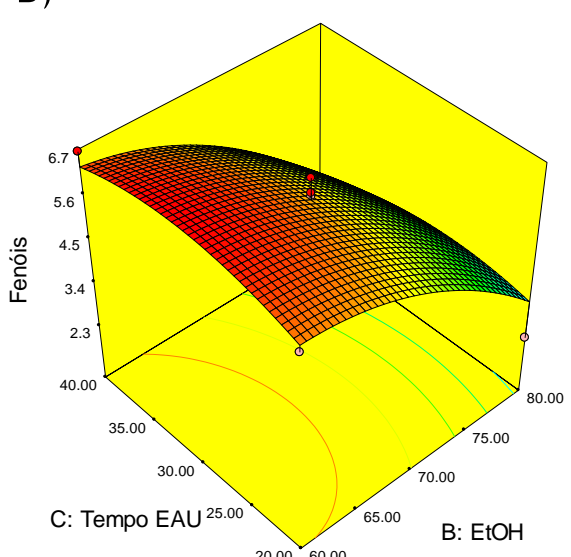

F)
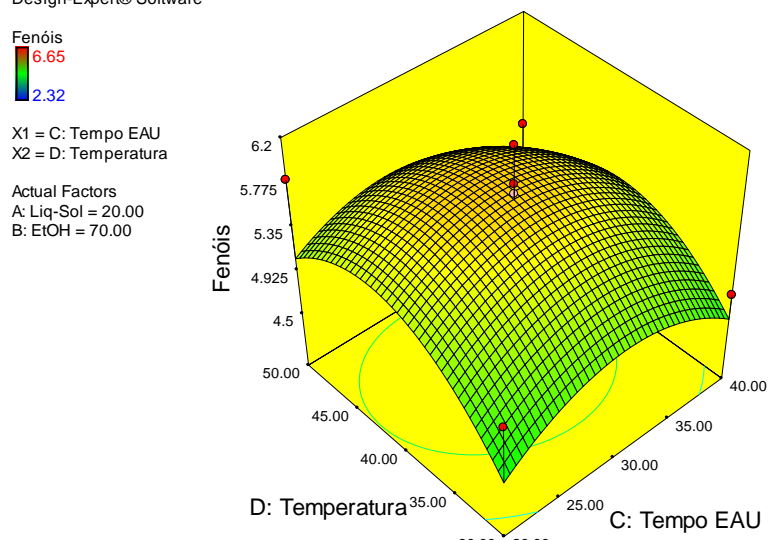

A) Razão de razão líquido-sólido e concentração de etanol, B) Razão líquido-sólido e tempo de EAU, C) Razão líquido-sólido e temperatura de EAU, D) Concentração de etanol e tempo de EAU, E) Concentração de etanol e temperatura de EAU e F) Tempo e temperatura de EAU como resposta o teor de fenóis. Fonte: Autores.

Na Figura 2C temos o efeito das variáveis temperatura e a relação líquido sólido mantendo constante a concentração de etanol a 70\% e o tempo de extração 30 min. A interação entre as variáveis não apresentou significância (P>0,05). Analisando o superfície de resposta, observa-se um diminuição no teor de fenólicos com o aumento da temperatura, este comportamento também é observado em relação líquido-sólido. O temparatura é um fator importante na extração de composto fenólicos, visto que influencia na permeabilidade das paredes celulares, aumentando a solubilidade e os coeficientes de difusão dos compostos 
a serem extraídos, entretanto em altas temperaturas, há um grande processo de degradação e/ou oxidação desses compostos (Santos-Buelga, et al., 2012).

Para a Figura 2D, observa-se o efeito das variáveis: concentração de etanol e tempo de EAU, mantendo constante a relação líquido-sólido a 20:1 e temperatura a $40^{\circ} \mathrm{C}$. A interação entre as variáveis não apresentou significância (P>0,05), e analisando o comportamento das variáveis, verificou que o tempo de extração (EAU) apresenta poucas alterações quando comparada à variável concentração de etanol. Contudo, temos que o ponto central foi de $30 \mathrm{~min}$, indicando o melhor resultado no processo de extração. Avaliando o efeito da concentração de etanol, observou-se que a diminuição no teor de etanol $(\mathrm{EtOH})$, proporcionou um aumento no teor de fenólicos obtidos.

O efeito da concentração de etanol e temperatura de EAU, mantendo constante a relação líquido-sólido 20:1 e o tempo de EAU 40 min é apresentada na Figura 2E. A interação dos efeitos entre estas variáveis mostrou não ser significativa ( $P>0,05)$. Apesar da interação entre essas variáveis não ser significativa, a extração dos compostos fenólicos é fortemente influenciada pela proporção de etanol no meio. Contudo, a variação da temperatura, na faixa estudada, não influenciou na extração.

A Figura 2F mostra a relação das variáveis temperatura e tempo de EAU, mantendo constante a relação líquido-sólido 20:1 e a concentração de etanol 70\%. A interação entre essas duas variáveis mostrou não ser significativa (P>0,05). Analisando a superfície de resposta (FIGD) observa-se um crescimento na obtenção de compostos fenólicos à medida que a temperatura aumenta de $30^{\circ} \mathrm{C}$ para $50^{\circ} \mathrm{C}$, após esse intervalo observa-se um decaimento, mostrando um ponto máximo entre $40,0^{\circ} \mathrm{C}$ e $45,0^{\circ} \mathrm{C}$. Em relação ao tempo de EAU, o mesmo comportamento é obtido, entretanto o ponto máximo deste apresenta-se em torno do ponto central 30,00 min.

Analisando os resultados provindos do planejamento fatorial Box-Behnken, pode-se verificar que as combinações de variáveis se apresentaram como não significativas diante dos resultados obtidos frente à otimização. Entretanto, sabe-se que todas as variáveis propostas nesse trabalho são importantes na extração dos compostos fenólicos de C. brasiliense, devido a cada variável utilizada nesse projeto ser de grande importância na extração de metabólitos presentes nas espécies botânicas. Diante da resposta obtida frente ao uso da ferramenta estatística, a variável que apresentou maior influência na eficiência extrativa dos compostos fenólicos, foi a concentração de etanol \% EtOH/ $\mathrm{H}_{2} \mathrm{O}$, este resultado está diretamente relacionado com os dados obtidos da Anova (tabela 4) e as superfícies de respostas.

A equação polinomial de segunda ordem (equação 3) expressa as condições otimizadas para extração dos compostos fenólicos da C. brasiliense: relação para líquida sólida de 18,7:1, concentração de etanol $60 \%$, tempo de EAU de 30 min e temperatura de $42{ }^{\circ} \mathrm{C}$. Sob essas condições foram extraídos $6,30 \mathrm{mg}(\mathrm{EAG}) \cdot \mathrm{g}^{-1}$ extrato $(\mathrm{n}=3)$, resultado que é aproximadamente $180 \%$ maior que o obtido nas condições iniciais (3,50 mg (EAG). $\mathrm{g}^{-1}$ extrato, L-S de 15:1, concentração de etanol $80 \%$, tempo de EAU de 50 min e temperatura de $40{ }^{\circ} \mathrm{C}$ ).

\section{Conclusão}

O planejamento fatorial Box-Behnken permitiu otimizar a extração de compostos fenólicos assistida por ultrassom e analisar quatro variáveis importantes para esse processo, dentre elas: relação líquido-sólido, concentração de etanol, tempo de extração assistida por ultrassom (EUA) e temperatura. Os resultados obtidos, apontaram que a variável mais importante no processo de extração dos compostos fenólicos da espécie C. brasiliense foi a concentração de etanol. Já as demais variáveis, não mostraram resultados significativos no processo de extração, apesar de serem variáveis importantes em um processo extrativo. Os resultados demostraram o sucesso e importância do processo de otimização com resposta aproximadamente 180 $\%$ maior que a obtida nas condições iniciais. 


\section{Agradecimentos}

Ao Conselho Nacional de Desenvolvimento Científico e Tecnológico (CNPq) pelo apoio financeiro (MCTI/CNPQ/Universal-14/2014/Processo: 453817/2014-9) e à Coordenadoria de Aperfeiçoamento de Pessoal de Nível Superior (CAPES).

\section{Referências}

Ahmad, A., Rehman, M. U., Wali, A. F., El-Serehy, H. A., Al-Misned, F. A., Maodaa, S. N., Aljawdah, H. M., Mir, T. M., \& Ahmad, P. (2020). BoxBehnken Response Surface Design of Polysaccharide Extraction from Rhododendron arboreum and the Evaluation of Its Antioxidant Potential. Molecules, 25(17). https://doi.org/10.3390/molecules25173835

Altemimi, A. B., Mohammed, M. J., Yi-Chen, L., Watson, D. G., Lakhssassi, N., Cacciola, F., \& Ibrahim, S. A. (2020). Optimization of ultrasonicated kaempferol extraction from ocimum basilicum using a box-behnken design and its densitometric validation. Foods, 9(10). https://doi.org/10.3390/foods9101379

Baptista, A., Gonçalves, R. V., Bressan, J., \& do Carmo Gouveia Pelúzio, M. (2018). Antioxidant and antimicrobial activities of crude extracts and fractions of cashew (Anacardium occidentale L.), cajui (Anacardium microcarpum), and pequi (Caryocar brasiliense C.): A systematic review. Oxidative Medicine and Cellular Longevity, 2018. https://doi.org/10.1155/2018/3753562

Bemfeito, C. M., Carneiro, J. de D. S., Carvalho, E. E. N., Coli, P. C., Pereira, R. C., \& Vilas Boas, E. V. de B. (2020). Nutritional and functional potential of pumpkin (Cucurbita moschata) pulp and pequi (Caryocar brasiliense Camb.) peel flours. Journal of Food Science and Technology, 57(10), 3920-3925. https://doi.org/10.1007/s13197-020-04590-4

Caldeira, A. S. P., Mbiakop, U. C., Pádua, R. M., van de Venter, M., Matsabisa, M. G., Campana, P. R. V., Cortes, S. F., \& Braga, F. C. (2021). Bioguided chemical characterization of pequi (Caryocar brasiliense) fruit peels towards an anti-diabetic activity. Food Chemistry, 345(November 2020), 1-10. https://doi.org/10.1016/j.foodchem.2020.128734

de Almeida, A. B., Silva, A. K. C., Lodete, A. R., Egea, M. B., Lima, M. C. P. M., \& Silva, F. G. (2019). Assessment of chemical and bioactive properties of native fruits from the Brazilian Cerrado. Nutrition and Food Science, 49(3), 381-392. https://doi.org/10.1108/NFS-07-2018-0199

de Oliveira, T. S., Thomaz, D. V., da Silva Neri, H. F., Cerqueira, L. B., Garcia, L. F., Gil, H. P. V., Pontarolo, R., Campos, F. R., Costa, E. A., dos Santos, F. C. A., de Souza Gil, E., \& Ghedini, P. C. (2018). Neuroprotective Effect of Caryocar brasiliense Camb. Leaves Is Associated with Anticholinesterase and Antioxidant Properties. Oxidative Medicine and Cellular Longevity, 2018, 1-12. https://doi.org/10.1155/2018/9842908

El-Naggar, N. E. A., Hamouda, R. A., Saddiq, A. A., \& Alkinani, M. H. (2021). Simultaneous bioremediation of cationic copper ions and anionic methyl orange azo dye by brown marine alga Fucus vesiculosus. Scientific Reports, 11(1), 1-19. https://doi.org/10.1038/s41598-021-82827-8

Ez Zoubi, Y., Fadil, M., Bousta, D., El Ouali Lalami, A., Lachkar, M., \& Farah, A. (2021). Ultrasound-Assisted Extraction of Phenolic Compounds from Moroccan Lavandula stoechas L.: Optimization Using Response Surface Methodology. Journal of Chemistry, 2021, 1-11. https://doi.org/10.1155/2021/8830902

Fonseca, L. D., Bastos, G. A., Costa, M. A. M. S., Ferreira, A. V. de P., Silva, M. L. F., Vieira, T. M., Morais-Costa, F., Oliveira, N. J. F. de, \& Duarte, E. R. (2018). Effects of Aqueous Extracts of Caryocar brasiliense in Mice. Acta Scientiae Veterinariae, 44(1), 6. https://doi.org/10.22456/1679-9216.80935

Ghitescu, R. E., Volf, I., Carausu, C., Bühlmann, A. M., Gilca, I. A., \& Popa, V. I. (2015). Optimization of ultrasound-assisted extraction of polyphenols from spruce wood bark. Ultrasonics Sonochemistry, 22, 535-541. https://doi.org/10.1016/j.ultsonch.2014.07.013

Güray, T., Menevşe, B., \& Yavuz, A. A. (2020). Determination of optimization parameters based on the Box-Behnken design for cloud point extraction of quinoline yellow using Brij 58 and application of this method to real samples. Spectrochimica Acta - Part A: Molecular and Biomolecular Spectroscopy, 243. https://doi.org/10.1016/j.saa.2020.118800

Jiang, Y., Wang, S., Yu, M., Wu, D., Lei, J., Li, W., He, Y., \& Gang, W. (2020). Ultrasonic-Assisted Ionic Liquid Extraction of Two Biflavonoids from Selaginella tamariscina. ACS Omega, 5(51), 33113-33124. https://doi.org/10.1021/acsomega.0c04723

Junior, A. J., Leitão, M. M., Bernal, L. P. T., dos Santos, E., Kuraoka-Oliveira, Â. M., Justi, P., Argandoña, E. J. S., \& Kassuya, C. A. L. (2019). Analgesic and Anti-inflammatory Effects of Caryocar brasiliense. Anti-Inflammatory \& Anti-Allergy Agents in Medicinal Chemistry, 19(3), 313-322. https://doi.org/10.2174/1871523018666190408144320

Lu, C. L., Zhu, Y. F., Hu, M. M., Wang, D. M., Xu, X. J., Lu, C. J., \& Zhu, W. (2015). Optimization of astilbin extraction from the rhizome of smilax glabra, and evaluation of its anti-inflammatory effect and probable underlying mechanism in lipopolysaccharide-induced raw264.7 macrophages. Molecules, 20(1), 625-644. https://doi.org/10.3390/molecules20010625

Moon, E. W., Lee, H. W., Rok, J. H., \& Ha, J. H. (2020). Photocatalytic inactivation of viral particles of human norovirus by Cu-doped TiO2 non-woven fabric under UVA-LED wavelengths. Science of the Total Environment, 749. https://doi.org/10.1016/j.scitotenv.2020.141574

Nam, S., Cho, H., Han, J., Her, N., \& Yoon, J. (2017). Photocatalytic degradation of acesulfame K : Optimization using the Box - Behnken design ( BBD ) Seong-Nam Nam a , c , Hyekyung Cho b , d, Jonghun Han c , Namguk Her c ,. Process Safety and Environmental Protection, 113, 10-21. https://doi.org/10.1016/j.psep.2017.09.002

Nascimento-Silva, N. R. R. D., \& Naves, M. M. V. (2019). Potential of Whole Pequi (Caryocar spp.) Fruit-Pulp, Almond, Oil, and Shell-as a Medicinal Food. Journal of Medicinal Food, 22(9), 952-962. https://doi.org/10.1089/jmf.2018.0149 
Nascimento-Silva, N. R. R., Mendes, N. S. R., \& Silva, F. A. (2020). Nutritional composition and total phenolic compounds content of pequi pulp (Caryocar brasiliense Cambess.). Journal of Bioenergy and Food Science, 07, 1-10. https://doi.org/10.18067/jbfs.v7i2.281

Neto, J. A., Tarôco, B. R. P., dos Santos, H. B., Thomé, R. G., Wolfram, E., \& Ribeiro, R. I. M. de A. (2020). Using the plants of Brazilian Cerrado for wound healing: From traditional use to scientific approach. Journal of Ethnopharmacology, 260(November 2018). https://doi.org/10.1016/j.jep.2020.112547

Oliveira, J. E., Martins, D. L., Dias, M. P. R., Treichel, T. L. E., \& Prado, T. D. (2020). Avaliação macroscópica da cicatrização de feridas de pele tratadas com extrato da folha de pequizeiro (Caryocar brasiliense). Brazilian Journal of Development, 6(4), 17649-17659. https://doi.org/10.34117/bjdv6n4-075

Pires, J., Cargnin, S. T., Costa, S. A., Sinhorin, V. D. G., Damazo, A. S., Sinhorin, A. P., Bicudo, R. de C., Cavalheiro, L., Valladão, D. M. de S., Pohlmann, A. R., Guterres, S. S., \& Ferrarini, S. R. (2020). Healing of dermal wounds property of Caryocar brasiliense oil loaded polymeric lipid-core nanocapsules: formulation and in vivo evaluation. European Journal of Pharmaceutical Sciences, 150(April). https://doi.org/10.1016/j.ejps.2020.105356

Prasad, K. N., Yang, E., Yi, C., Zhao, M., \& Jiang, Y. (2009). Effects of high pressure extraction on the extraction yield, total phenolic content and antioxidant activity of longan fruit pericarp. Innovative Food Science and Emerging Technologies, 10(2), 155-159. https://doi.org/10.1016/j.ifset.2008.11.007

Ramos, R. de O., Pertuzatti, P. B., Gomes, I. M., Santana, M. B., Brito, R. de M., Tussolini, M., Miguel, T. B., \& Tussolini, L. (2021). Chemical and antioxidant characterization, sensory and shelf-life analysis of cereal bars with almonds from pequi (Caryocar brasiliense camb.). Food Science and Technology (Brazil), 41(June), 368-374. https://doi.org/10.1590/fst.29218

Santos-Buelga, C., Gonzalez-Manzano, S., Dueñas, M., \& Gonzalez-Paramas, A. M. (2012). Extraction and isolation of phenolic compounds. In Methods in Molecular Biology (Vol. 864). https://doi.org/10.1007/978-1-61779-624-1_17

Singleton, V. L., Orthofer, R., \& Lamuela-Raventós, R. M. (1999). Analysis of Total Phenols and Other Oxidation Substrates and Antioxidants by Means of Folin-Ciocalteu Reagent. METHODS IN ENZYMOLOGY, 299(1974), 152-178. https://doi.org/10.1016/s0076-6879(99)99017-1

Swartz, M. R. \& Krull, I. S. (1998). Validação de Métodos Cromatográficos. Pharmaceutical Technology, São Paulo, 2 (1), $12-20$.

Valu, M.-V., Soare, L. C., Sutan, N. A., Ducu, C., Moga, S., Hritcu, L., Boiangiu, R. S., \& Carradori, S. (2020). Optimization of Ultrasonic Extraction to Obtain Erinacine A and Polyphenols with Antioxidant Activity from the Fungal Biomass of Hericium erinaceus. Foods, 9(12), 1889. https://doi.org/10.3390/foods9121889

Vizzotto, M., \& Pereira, M. C. (2011). Amora-preta (Rubus sp.): Otimização do processo de extração para determinação de compostos fenólicos antioxidantes. Revista Brasileira de Fruticultura, 33(4), 1209-1214. https://doi.org/10.1590/S0100-29452011000400020

Yuan, Z., Xu, Z., Zhang, D., Chen, W., Zhang, T., Huang, Y., Gu, L., Deng, H., \& Tian, D. (2018). Box-Behnken design approach towards optimization of activated carbon synthesized by co-pyrolysis of waste polyester textiles and $\mathrm{MgCl} 2$. Applied Surface Science, 427, 340-348. https://doi.org/10.1016/j.apsusc.2017.08.241

Zhang, H., Yan, H., Li, Q., Lin, H., \& Wen, X. (2021). Identification of VOCs in essential oils extracted using ultrasound- and microwave-assisted methods from sweet cherry flower. Scientific Reports, 11(1), 1-13. https://doi.org/10.1038/s41598-020-80891-0 